\title{
Pressure-temperature phase behavior of mixtures of natural sphingomyelin and ceramide extracts
}

Hanna M. G. Barriga, Edward S. Parsons, Nicola L. C. McCarthy, Oscar Ces, John M. Seddon, Robert V. Law and Nicholas J. Brooks*

Department of Chemistry, Imperial College London, South Kensington, London, SW7 2AZ, UK

\begin{abstract}
Ceramides are a group of sphingolipids that act as highly important signaling molecules in a variety of cellular processes including differentiation and apoptosis. The predominant in-vivo synthetic pathway for ceramide formation is via sphingomyelinase catalyzed hydrolysis of sphingomyelin. The biochemistry of this essential pathway has been studied in detail, however there is currently a lack of information on the structural behavior of sphingomyelin and ceramide rich model membrane systems, which is essential for developing a bottom up understanding of ceramide signaling and platform formation. We have studied the lyotropic phase behavior of sphingomyelin - ceramide mixtures in excess water as a function of temperature $\left(30-70{ }^{\circ} \mathrm{C}\right)$ and pressure $(1-200 \mathrm{MPa})$ by small- and wide-angle X-ray scattering. At low ceramide concentrations the mixtures form the ripple gel phase $\left(\mathrm{P}_{\beta}{ }^{\prime}\right)$ below the gel transition temperature for sphingomyelin and this observation has been confirmed by atomic force microscopy. Formation of the ripple gel phase can also be induced at higher temperatures via the application
\end{abstract}


of hydrostatic pressure. At high ceramide concentration an inverse hexagonal phase $\left(\mathrm{H}_{\mathrm{II}}\right)$ is formed coexisting with a cubic phase.

\section{INTRODUCTION}

Ceramides are a group of sphingolipids based on a sphingosine backbone with a fatty acid chain attached via an amide linkage. Their molecular structure is closely related to that of sphingomyelin (SM) (Figure 1) and there are a number of different pathways for their synthesis in-vivo. ${ }^{1}$ The formation of ceramides via the catalyzed hydrolysis of sphingomyelin is a rapid, localized event and thought to be particularly important in relation to apoptosis which is known to be accompanied by an increase in ceramide concentrations. Ceramides are also thought to act as important signaling molecules in a range of biological processes, however the mechanisms that underpin this are not well understood..$^{2}$ In addition, recent work has also indicated that increased ceramide concentrations in the brain can be linked to depression. ${ }^{3}$

Ceramide aggregation in biomembranes is thought to play a key role in certain signaling pathways; sphingolipid rich microdomains in plasma membranes have been associated with trans-membrane signaling events and there is also significant evidence that modulation of the local membrane curvature and ordering by ceramide aggregation is essential to biological function. ${ }^{1}$ The main transition temperature for long $\mathrm{N}$-acyl chain ceramides is extremely high $\left(\sim 70^{\circ} \mathrm{C}\right)$ and so ceramide rich membrane domains tend to be highly ordered with significantly reduced lateral diffusion which must have a significant effect on both the membrane and biomolecules embedded within it. ${ }^{4}$ Despite its biological importance, there is very little physicochemical data probing the interaction between sphingomyelin and ceramides. Understanding this and the mechanism by which they can modulate membrane structure is key to fully understanding the critical role that ceramides play in biology. 
We have begun to address this by studying the pressure-temperature evolution of small and wide angle X-ray diffraction (SAXS / WAXS) patterns in model membranes containing ceramide and sphingomyelin. By generating model membranes with different ratios of ceramide and sphingomyelin, we aim to mimic the concentrations of these components that may occur during sphingomyelinase activity on biological membranes, as well as probing the extremes that could occur locally during ceramide aggregation.

In this work, natural SM and ceramide extracts from chicken egg (ESM and EC respectively) were used. The amide linked hydrocarbon chain in the major component ( $>84 \mathrm{~mol} \%)$ of both of these extracts is 16 carbon atoms long and fully saturated (16:0) (Figure 1). Previous characterizations of both ESM and EC have shown that the remaining species are mixtures with chain lengths varying from 14:0 to $24: 0 .^{5}$

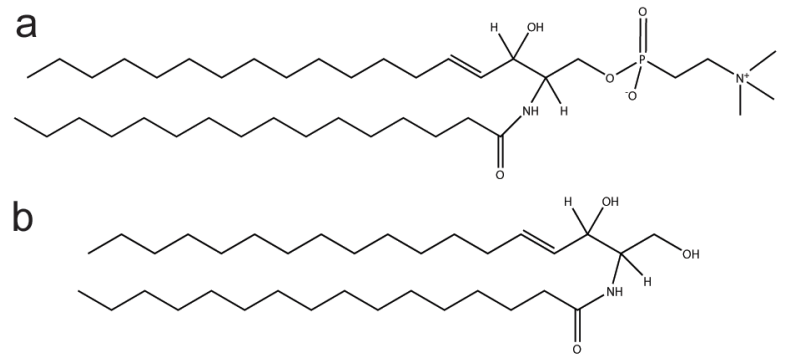

Figure 1. Structure of (a) 16:0 sphingomyelin (b) 16:0 ceramide, which are the major components of sphingomyelin and ceramide extracts from egg.

Both ESM and pure 16:0 ceramide have been studied previously by differential scanning calorimetry; $;^{4,6}$ the transition temperatures are summarized in Table 1. 


\begin{tabular}{lll}
\hline Lipid & $\begin{array}{l}\text { Transition } \\
\text { temperature } /{ }^{\circ} \mathbf{C}\end{array}$ & Transition type \\
\hline ESM & $37-40$ & Gel - fluid \\
Ceramide (16:0) & 68 & Metastable - stable gel \\
Ceramide (16:0) & 91 & Gel - fluid \\
\hline
\end{tabular}

Table 1. Transition temperatures for ESM and 16:0 ceramide.

Previous physico-chemical studies of natural extract sphingomyelins in excess water have shown that ESM (and also bovine brain sphingomyelin) can form a ripple gel phase at temperatures below the main gel-fluid transition. ${ }^{7}$ In particular, ESM exhibits a ripple phase in between the unrippled lamellar gel phase and the fluid lamellar phase, with a narrow coexistence region between the fluid lamellar and ripple gel phases.

Synthetic 16:0 ceramide in excess water is known to undergo a number of phase transitions; below $68^{\circ} \mathrm{C}, \mathrm{X}$-Ray diffraction studies have shown that it adopts a lamellar structure that gives a single WAXS reflection, attributed to the presence of a metastable gel state. Above $68^{\circ} \mathrm{C}$, the lamellar geometry remains, however there is a small change in the lattice parameter and the WAXS now shows a number of different reflections which are indicative of the thermodynamically stable structure (often referred to as the 'stable state'). ${ }^{4}$

Previous studies have also shown the tendency of ceramides to modify phase transition temperatures when mixed with other lipids and drive the formation of non-lamellar (type II) phases ${ }^{8,9,10}$ It has been demonstrated by DSC that when ceramide is incorporated into the phospholipid dielaidoylphosphatidylethanolamine (DEPE) ${ }^{10}$ at concentrations of over $25 \mathrm{~mol} \%$, the gel - fluid transition temperature is increased by approximately $8{ }^{\circ} \mathrm{C}$ and occurs over a wide temperature range $\left(\sim 15^{\circ} \mathrm{C}\right)$. In addition, the lamellar to hexagonal phase transition temperature of DEPE was also reduced and the transition broadened by the addition of ceramide. Similar 
effects on the gel - fluid transition temperature have been observed when ceramide is added to dipalmitoylphosphatidylcholine (DPPC).

Some data already exists on the behavior and interactions of ceramides with different lipids, including for different chain length sphingomyelins and ceramides. ${ }^{11,12,13,14}$ In particular there are many studies using ceramides to probe domain formation in vesicular systems. Giant unilamellar vesicles (GUVs) containing mixtures of ESM and EC have been reported to show domains when doped with a fluorescent dye that selectively partitions into liquid disordered domains. ${ }^{15}$ Above $40{ }^{\circ} \mathrm{C}, \mathrm{SM}$ rich domains are preferentially solubilized over ceramide rich ones when treated with detergent; an observation that may be relevant to some detergent resistant cell membranes. Similar domain formation has also been seen in mixtures of cholesterol phosphocholine (CholPC) $)^{13}$ and ceramides as well as in vesicles composed of phospholipid, ceramide and cholesterol. ${ }^{16}$ Interestingly, SAXS and electron spin resonance (ESR) spectroscopy studies have shown a competition for interaction with sphingomyelin between ceramide and cholesterol, ${ }^{17}$ as well as evidence suggesting that ceramide can displace cholesterol in liquid ordered $\left(\mathrm{L}_{\mathrm{o}}\right)$ domains ${ }^{18}$ and increase the order parameter of the $\mathrm{L}_{\mathrm{o}}$ phase. ${ }^{19}$

In this study we use pressure and temperature to map the phase behavior of ESM : EC mixtures. We show that at low EC concentrations, the ESM ripple is maintained, but rapidly disrupted at higher concentrations. At high temperatures and high EC content, EC is observed to promote non lamellar phases in ESM, typically a lamellar phase forming lipid. In addition we use AFM to confirm the presence of the ripple phase in mixtures and DSC to verify the transitions shown in pressure-temperature diagrams. 


\section{EXPERIMENTAL METHODS}

\section{Materials}

Egg sphingomyelin (ESM) and egg ceramide (EC) were purchased from Avanti Polar Lipids (Alabaster, AL, USA) as a lyophilised powder with a purity of $>99 \%$ and were used without further purification. To ensure the lipids were completely dry they were lyophilised (Thermo Scientific, Asheviller, NC, USA) for a further 12 hours before use. Lipid mixtures were prepared by co-dissolving appropriate masses of ESM and EC in chloroform (Sigma Aldrich, Gillingham, Dorset, UK). These mixtures were dried under a stream of nitrogen gas for 1 hour and then lyophilised for a minimum of 12 hours after which they were sealed and stored at $-20{ }^{\circ} \mathrm{C}$.

\section{Small and wide angle X-ray diffraction (SAXS / WAXS)}

Sample mixtures were hydrated with MilliQ water to $70 \mathrm{wt} \%$. They were then sealed and heat cycled (between -196 and $90{ }^{\circ} \mathrm{C}$ ) 20 times to promote mixing. SAXS / WAXS data was obtained between $30-70{ }^{\circ} \mathrm{C}$ (at $10^{\circ} \mathrm{C}$ intervals), and $1-200 \mathrm{MPa}$ (at $40 \mathrm{MPa}$ intervals) at beamline I22, Diamond Light Source using a custom developed high pressure sample environment, ${ }^{20}$ an X-ray energy of $18 \mathrm{keV}$ and a sample to detector distance of $1.2 \mathrm{~m}$. The length scale range $(3-300 \AA)$ accessible on the large format Pilatus 2M detector enabled simultaneous and continuous capture of SAXS and WAXS data. The upper temperature limit studied is defined by the maximum stable temperature obtainable with the high pressure system. Images were analyzed using the inhouse developed software package AXcess. ${ }^{21}$ Briefly, the two dimensional SAXS patterns were radially integrated to give one dimensional diffraction patterns. The Bragg peaks were then fitted using Gaussian functions and indexed by comparison to characteristic diffraction patterns from known lipid structures. 


\section{Atomic Force Microscopy (AFM)}

Supported lipid bilayers were prepared by hydrating a dried lipid film to a final concentration of $1 \mathrm{mg} / \mathrm{ml}$ in MilliQ water, which was then bath sonicated for approximately $1 \mathrm{~h}$ until clear. The resulting small unilamellar vesicle suspension $(60 \mu 1)$ was incubated over freshly cleaved mica at $60{ }^{\circ} \mathrm{C}$ for 45 minutes resulting in fusion of the lipid vesicles with the surface. Excess vesicles were removed with gentle washing with MilliQ water $(100 \mu 1)$, thus leaving a secondary (or floating) supported lipid bilayer. This is a double bilayer and the top bilayer is visualized as described previously by Leidy et al. ${ }^{22}$ Samples were loaded into a Veeco Multimode IV AFM and images were acquired in tapping mode using Nanosensors PNP-TR cantilevers. Care was taken through the experiments to ensure that the supported bilayers remained fully hydrated by depositing a drop of water on top of the sample..

\section{Differential Scanning Calorimetry (DSC)}

Dry lipid mixtures were loaded into DSC sample pans (Aluminium Hermetic, TA instruments, Elstree, UK), hydrated to 70 wt\% with MilliQ water and sealed. DSC thermograms were collected using a Perkin-Elmer Diamond DSC over a temperature range of $10-90{ }^{\circ} \mathrm{C}$ with a heating / cooling rate of $15{ }^{\circ} \mathrm{C} / \mathrm{min}$. Similar (but reversed) transitions were observed in the heating and cooling scans. Data was analyzed using the Origin data analysis package. 


\section{RESULTS AND DISCUSSION}

The phase behavior of five ESM : EC mixtures were mapped with lipid ratios from $90: 10$ mol\% to 40 : $60 \mathrm{~mol} \%$ (Figure 2) using data obtained from SAXS/WAXS (0-200 MPa) and DSC (atmospheric pressure) measurements (ESI Figure S2). For clarity only the phase boundaries have been shown here, however datapoints are included in the ESI Figure S1. Over the composition, pressure and temperature range mapped, six distinct regions were observed. These include two flat gel phases $\left(\mathrm{L}_{\beta} 1\right.$ and $\left.\mathrm{L}_{\beta} 2\right)$, a ripple gel phase $\left(\mathrm{P}_{\beta}{ }^{\prime}\right)$, similar to that previously observed in ESM, a fluid lamellar phase $\left(\mathrm{L}_{\alpha}\right)$ and region of hexagonal $\left(\mathrm{H}_{\mathrm{II}}\right) /$ cubic phase coexistence. These have been shown schematically in Figure 3. 
a

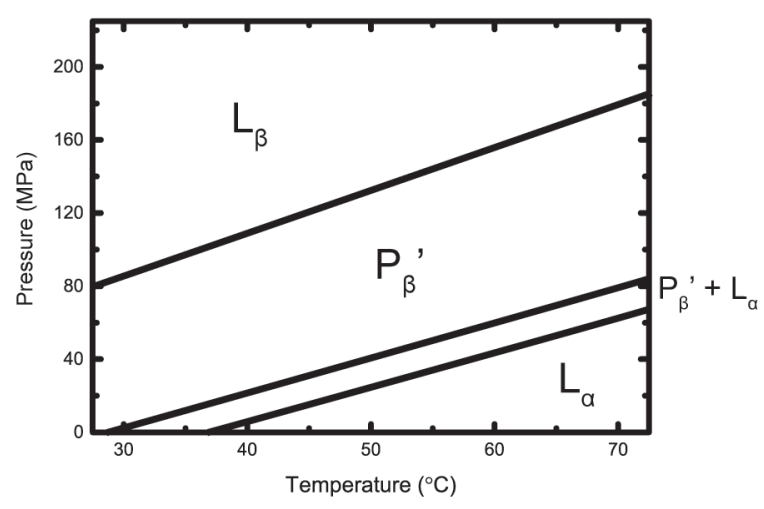

C

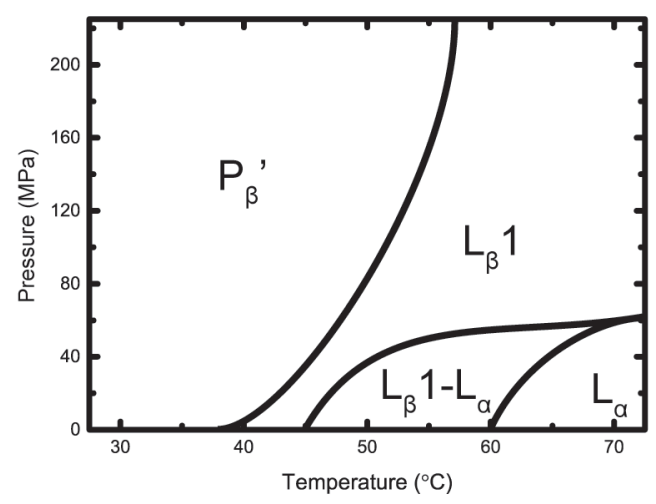

e

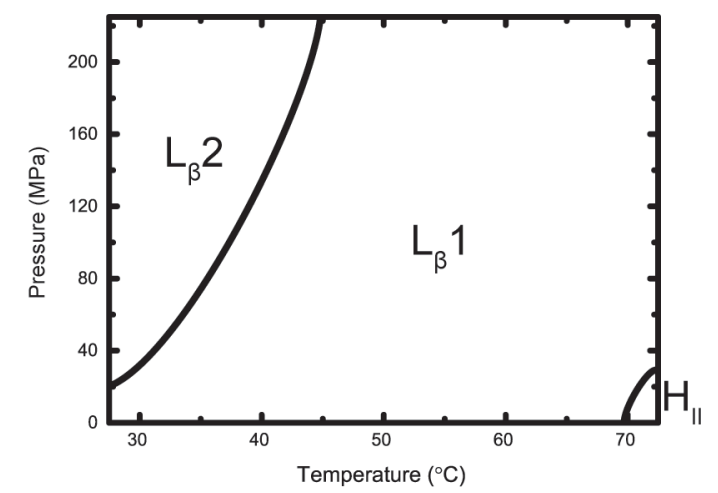

b

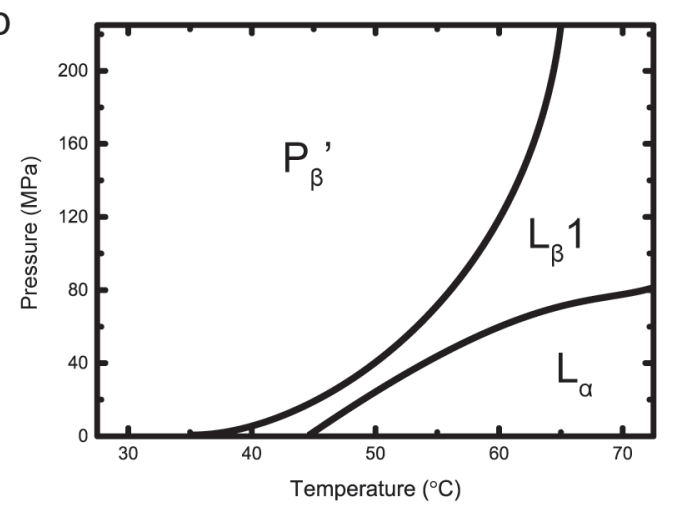

d
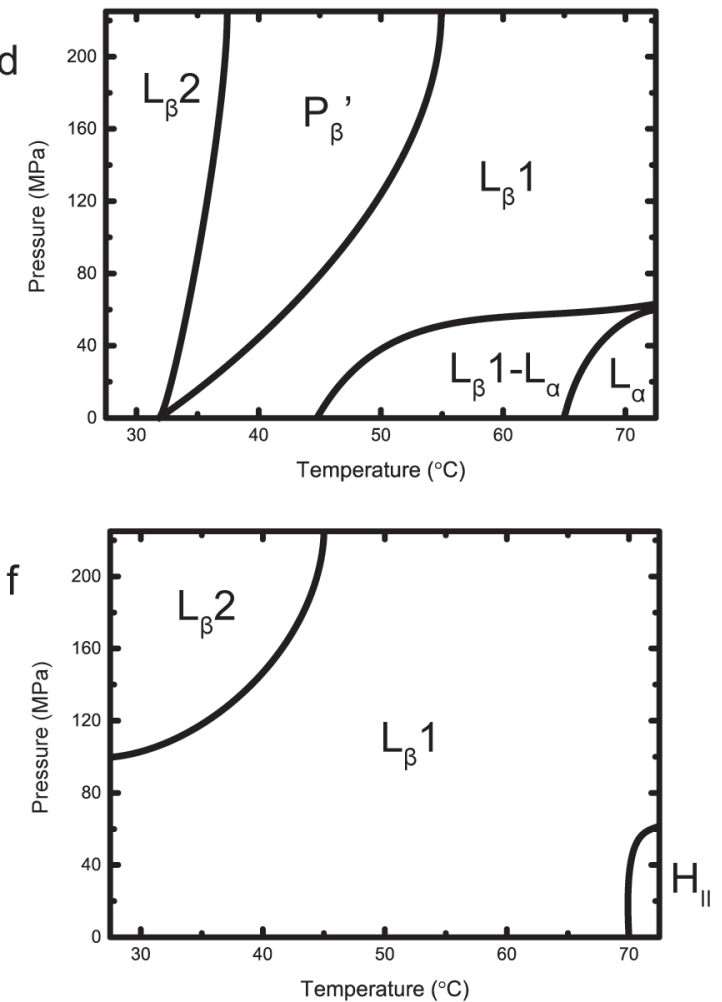

Figure 2. Pressure-temperature diagrams showing the phase behavior of ESM : EC mixtures (a) 100:0 (b) 90:10 (c) 80:20 (d) 70:30 (e) 60:40 (f) 40:60 mol\%, where (a) is adapted from Shaw et al. ${ }^{7}$ Data was obtained from SAXS/WAXS (0-200 MPa) and DSC (atmospheric pressure) measurements. The region marked $\mathrm{H}_{\mathrm{II}}$ exhibits coexistence between a hexagonal and cubic phase. It should be noted that there is likely to be small coexistence regions at the phase 
transition boundaries, but these were below the pressure and temperature resolution of the experiment.
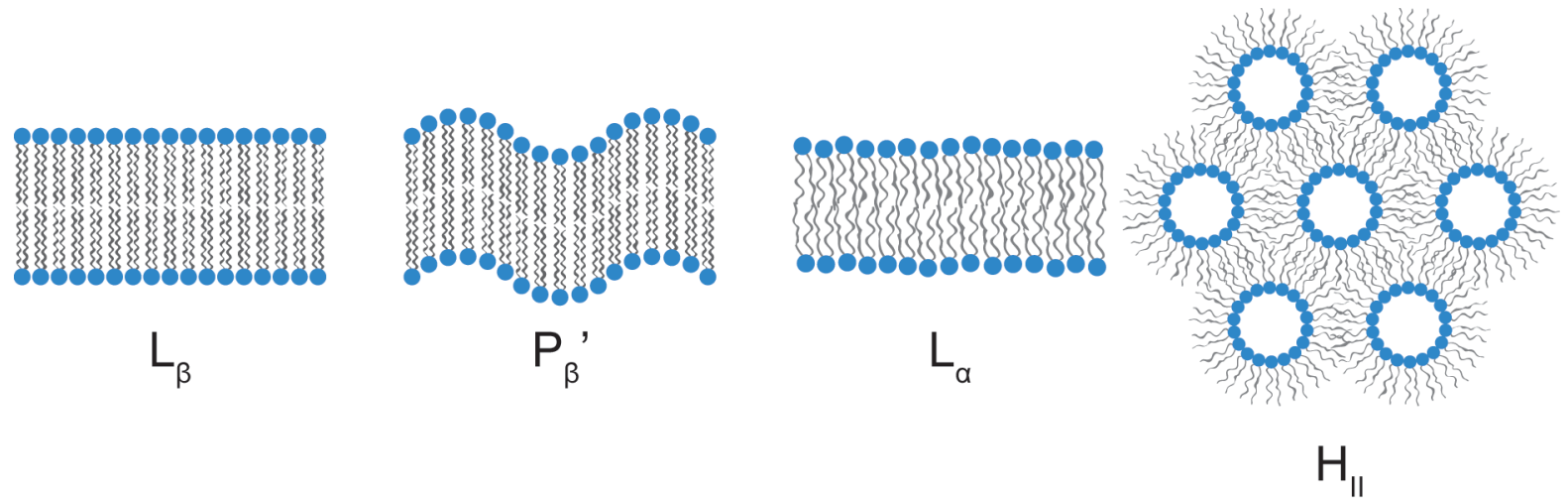

Figure 3. Schematic illustrations of the different phases observed in the ESM:EC phase diagram including the gel phase $\left(\mathrm{L}_{\beta}\right)$, ripple phase $\left(\mathrm{P}_{\beta}{ }^{\prime}\right)$, fluid lamellar $\left(\mathrm{L}_{\alpha}\right)$ and the hexagonal phase $\left(\mathrm{H}_{\mathrm{II}}\right)$.

DSC thermograms were collected for sample mixtures ranging from pure ESM to EC at atmospheric pressure to confirm the positions of the phase boundaries found during the SAXS experiments. The fitted results are summarized in Table 2 and show good agreement with both $\mathrm{X}$-ray data collected at atmospheric pressure and literature values for pure $\mathrm{ESM}^{7,11}$ and $\mathrm{EC}^{4,11}$.

\begin{tabular}{ccccccc}
\hline $\begin{array}{c}\text { Composition } \\
\text { (ESM:EC mol\%) }\end{array}$ & $\begin{array}{c}\text { Transition 1 } \\
\text { Onset } \\
\left({ }^{\circ} \mathbf{C}\right)\end{array}$ & $\begin{array}{c}\text { FWHM } \\
\left({ }^{\circ} \mathbf{C}\right)\end{array}$ & Type & $\begin{array}{c}\text { Transition 2 } \\
\text { Onset } \\
\left({ }^{\circ} \mathbf{C}\right)\end{array}$ & $\begin{array}{c}\text { FWHM } \\
\left({ }^{\circ} \mathbf{C}\right)\end{array}$ & Type \\
\hline $100: 0$ & 39.3 & 3.3 & $\mathrm{P}_{\beta}{ }^{\prime}-\mathrm{L}_{\alpha}$ & - & - & - \\
$80: 20$ & 38.6 & 3.7 & $\mathrm{P}_{\beta}{ }^{\prime}-\mathrm{L}_{\beta} 1$ & 66.3 & 4.9 & $\mathrm{~L}_{\beta} 1-\mathrm{L}_{\alpha}$ \\
$60: 40$ & 69.3 & 4.6 & $\mathrm{~L}_{\beta} 1-\mathrm{H}_{\mathrm{II}}$ & - & - & - \\
$40: 60$ & 71.4 & 4.7 & $\mathrm{~L}_{\beta} 1-\mathrm{H}_{\mathrm{II}}$ & - & - & - \\
$0: 100$ & 68.7 & 2.7 & $\begin{array}{c}\text { Metastable- } \\
\text { Stable Gel }\end{array}$ & 83.9 & 4.2 & Gel-Fluid \\
\hline
\end{tabular}

Table 2. Phase transition temperatures (Onset) of ESM : EC mixtures from DSC thermograms performed at atmospheric pressure, the full width half maximum (FWHM) of the transition peak, and the starting and ending phases (Type). 


\section{Ripple phase $\left({ }_{\beta}{ }^{\prime}\right)$}

At low ceramide concentrations and below the gel-fluid transition temperature for pure ESM, the mixtures adopt a ripple phase similar to that previously observed in pure $\operatorname{ESM}^{7}$ (Figure 4a). As previously observed for pure ESM, diffraction patterns from the ripple phase of ESM rich mixtures are less well resolved than for other sphingomyelin extracts such as that from bovine brain, ${ }^{7}$ however the characteristic peak envelope and low intensity, small angle peak (at around $0.0065 \AA^{-1}$ in Figure 4a) strongly suggest ripple phase formation. Due to the poor resolution, accurate lattice parameter determination is not possible, however we have been able to estimate these by assuming $\gamma$ (the angle between the a and b lattice parameters) is $90^{\circ}$ as previously, ${ }^{7} \mathrm{a}$ (the spacing between the center of one bilayer and the next) is then given by the $\mathrm{d}$ spacing of the 10 peak which is assigned as the high intensity peak at approximately $0.015 \AA^{-1}$ and $\mathrm{b}$ (the ripple period) is given by the $\mathrm{d}$ spacing of the 01 peak which is the small peak at approximately 0.0065 $\AA^{-1}$. These estimated parameters show an increase of approximately $2 \AA$ in layer spacing with increasing EC content, from a pure ESM layer spacing of approximately $67 \AA$. In comparison, the flat $\mathrm{L}_{\beta}$ gel phases show sharp, evenly spaced diffraction peaks as expected (Figure $4 \mathrm{~b}$ ). The lattice parameter of the $L_{\beta}$ phase shown in Figure $4 b$ is approximately $1.5 \AA$ lower than that of the $\mathrm{P}_{\beta}$ phase shown in Figure 4a, possibly due to the higher EC content or due a tilting of the chains in the $\mathrm{P}_{\beta}$ phase. 

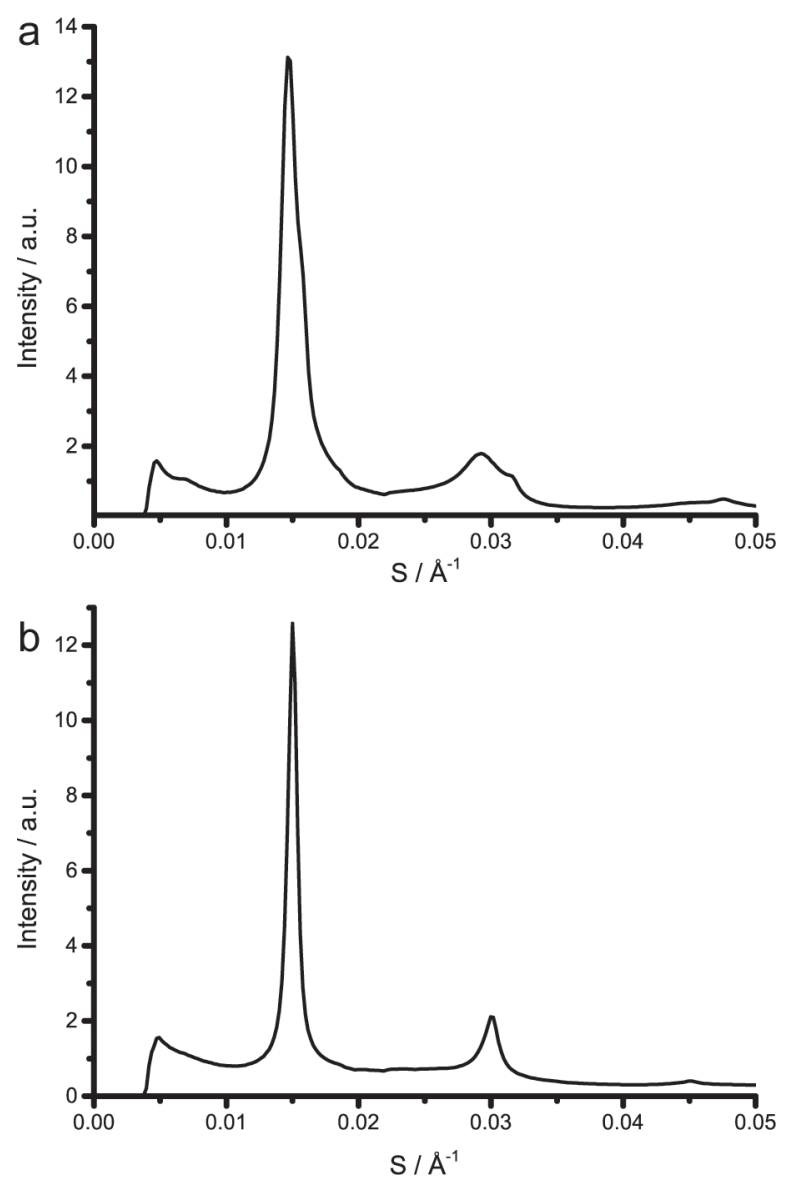

Figure 4. SAXS patterns from (a) the ripple gel phase $\left(\mathrm{P}_{\beta}{ }^{\prime}\right)$ formed by ESM:EC 90:10 mol\% at $30{ }^{\circ} \mathrm{C}$ and atmospheric pressure and (b) the flat gel phase $\left(\mathrm{L}_{\beta} 1\right)$ formed by ESM:EC 80:20 mol\% at $60{ }^{\circ} \mathrm{C}$ and $80 \mathrm{MPa}$.

In pure ESM, DSC data shows a single peak with an onset of $39.3{ }^{\circ} \mathrm{C}$ (ESI Figure S2) corresponding to the ripple to fluid lamellar transition as previously reported. ${ }^{7}$ After addition of up to $20 \mathrm{~mol} \% \mathrm{EC}$, the ripple phase is still visible, and it shows a similar transition temperature however, SAXS data shows that the ripple phase no longer transforms to a fluid lamellar phase but to a flat gel phase. 
The presence of the ripple phase at low ceramide concentrations implies that EC is, to a limited extent, miscible with the ESM gel phase. Previous reports of EC mixed with the phospholipids $\mathrm{DEPE}^{9}$ and $\mathrm{DPPC}^{23}$ have reported the coexistence of two gel phases rather than a single gel ripple phase as observed here, suggesting that this miscibility is facilitated by the specific structural and hydrogen bonding interactions that are possible between sphingomyelin and ceramide as a result of their closely related molecular structures.$^{24}$ In addition, we have observed the ripple phase and the dissolving of EC in the predominantly ESM membrane using AFM in pure ESM and a ESM:EC 80:20 mol\% mixture (the highest concentration of EC seen to adopt the ripple phase at atmospheric pressure). A clear ripple structure can be seen in both samples (Figure 5) with a ripple period of approximately $130 \AA$ for ESM (which agrees well with previous SAXS data $^{7}$ ) and $330 \AA$ for the ESM:EC mixture, indicating that the addition of EC disrupts the ripple phase, increasing the ripple period. The AFM results show some heterogeneity in the sample and we believe that the more flattened topography represents a small amount of $\mathrm{L}_{\beta}$ impurity as previously observed in bovine brain sphingomyelin. The AFM measurements are significantly more sensitive to heterogeneity within the sample compared to SAXS so the signal from this $\mathrm{L}_{\beta}$ impurity would be swamped by the predominant ripple phase signal in the X-ray measurements. The increase in the ripple period on addition of EC in the AFM data and loss of the ripple in favor of a flat gel phase by $30 \mathrm{~mol} \%$ EC suggests the gradual loss of the ripple by a 'stretching out' mechanism as previously observed during the melting of bovine brain sphingomyelin. ${ }^{7}$ 

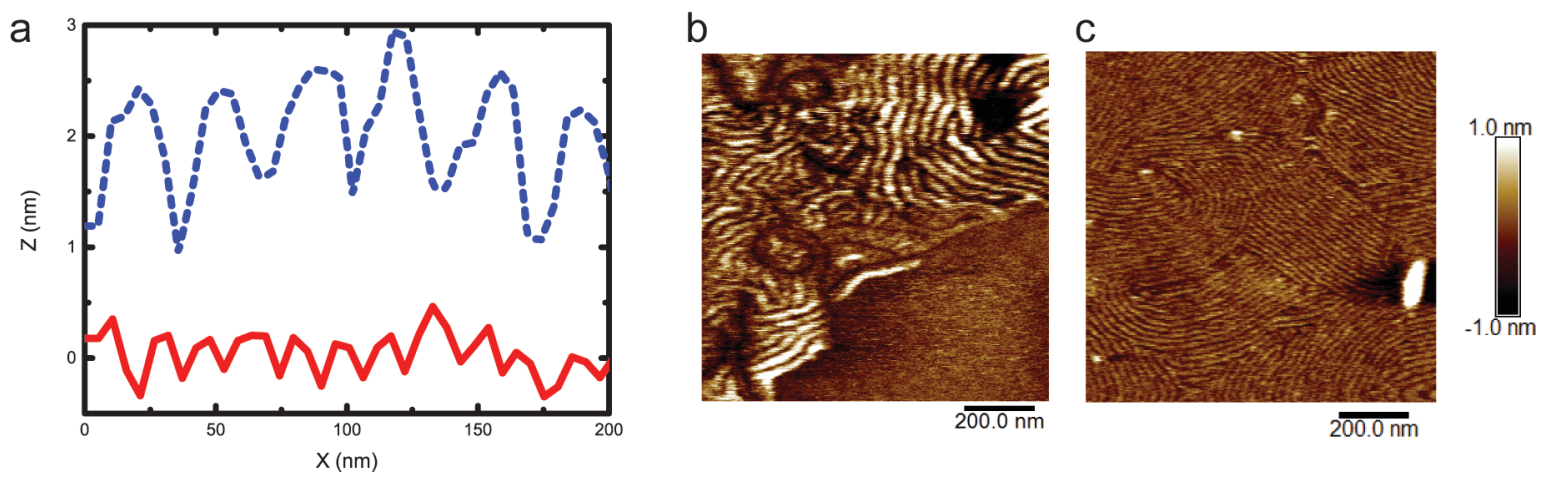

Figure 5. AFM data from ESM:EC mixtures showing (a) the ripple period of ESM (solid, red) and ESM:EC 80:20 mol\% (dashed, blue) (b) ESM:EC 80:20 mol\% image (c) ESM:EC 100:0 mol\% image. Note that the observed increase in ripple amplitude is likely to be an artifact of the imaging process, whereby the AFM tip (with a nominal tip radius of $10 \mathrm{~nm}$ ) is unable to penetrate fully into the troughs of the ripple for pure SM (ripple period $13 \mathrm{~nm}$ ) and thus gives a lower value of ripple amplitude.

Of particular interest is the induction of the ripple phase by application of pressure. At atmospheric pressure, the ripple phase is only visible up to $20 \mathrm{~mol} \% \mathrm{EC}$ as shown in both the SAXS and AFM data. However, when pressure is applied to the sample containing $30 \mathrm{~mol} \% \mathrm{EC}$, the ripple phase forms at $80 \mathrm{MPa}$. Pressure will always drive formation of lower volume structures $^{25}$ and so this suggests that the ripple phase in these mixtures is a more compact structure than the flat gel phase. In the case of bovine brain sphingomyelin, hydrostatic pressure has been shown to significantly sharpen the SAXS pattern observed from the ripple phase. ${ }^{7}$ A slight decrease in lattice parameter of up to $1 \AA$ is seen in the ripple phase in both 80:20 and 70:30 ESM:EC mixtures over a pressure increase of $200 \mathrm{MPa}$. In previous investigations, bovine brain has also shown little change in lattice parameter with changes in hydrostatic pressure. ${ }^{7}$ 


\section{$L_{\beta}$ gel phases $\left(L_{\beta} 1\right.$ and $\left.L_{\beta} 2\right)$}

Whilst the addition of up to $20 \mathrm{~mol} \% \mathrm{EC}$ to ESM does not significantly disrupt the formation of the ripple phase at atmospheric pressure, it does affect the transition from the ripple to the fluid phase observed in pure ESM. Instead this transition is replaced by a transition from a ripple to a gel phase $\left(\mathrm{L}_{\beta} 1\right)$ at the ESM transition temperature. This gel phase is characterized by a single strong peak in the WAXS pattern. This wide angle peak is very similar to that observed in pure EC in which it is attributed to the tilted metastable gel state. ${ }^{4}$ Notably, the DSC peak corresponding to the $\mathrm{P}_{\beta}{ }_{\beta}$ to $\mathrm{L}_{\beta} 1$ transition in $20 \mathrm{~mol} \% \mathrm{EC}$ is similar in magnitude to that of the $\mathrm{L}_{\beta} 1$ to $\mathrm{L}_{\alpha}$ transition suggesting that the inter-gel transition is accompanied by a significant conformational change as shown in the ESI (Figure S2b). We believe that the $\mathrm{L}_{\beta} 1$ phase is closely structurally related to the EC tilted metastable gel state and indeed the $\mathrm{L}_{\beta} 1$ region of the pressure - temperature phase diagram expands significantly with increasing EC concentration (Figure 2).

While previous DSC investigations ${ }^{14}$ have attributed the transition observed in low EC concentration mixtures at around the pure ESM main transition temperature solely to an $\mathrm{L}_{\beta}-\mathrm{L}_{\alpha}$ coexistence region, the SAXS data obtained here show a single lamellar phase above this temperature (ESI Figure S3a), which we have attributed to the $\mathrm{L}_{\beta} 1$ gel phase. However, at higher temperatures we do observe coexistence between fluid and gel phases (ESI Figure S3b). We cannot completely exclude the possibility that the $\mathrm{L}_{\beta} 1$ gel phase coexists with a fluid phase that has an identical lattice parameter, however based on the other data gathered here, this seems unlikely.

At $30 \mathrm{~mol} \% \mathrm{EC}$ and above, two distinct flat gel phases were observed. In the $30 \mathrm{~mol} \% \mathrm{EC}$ sample, at atmospheric pressure and low temperatures, the ripple phase is replaced by a single 
flat $\mathrm{L}_{\beta}$ gel phase. This $\mathrm{L}_{\beta}$ gel phase (referred to as $\mathrm{L}_{\beta} 2$ in Figure 2) is distinguished from $\mathrm{L}_{\beta} 1$ in that it displays a series of poorly resolved broad peaks in the WAXS pattern rather than a strong single peak. ${ }^{4}$ It is likely that below the main transition temperature for ESM, when both the ESM and EC preferentially adopt a gel state, strong interactions between the sphingomyelin and ceramide (as noted above) cause formation of a single phase but that the packing of the EC is sufficiently disrupted to avoid formation of its metastable state. Examples of both gel states have been shown in Figure 6.
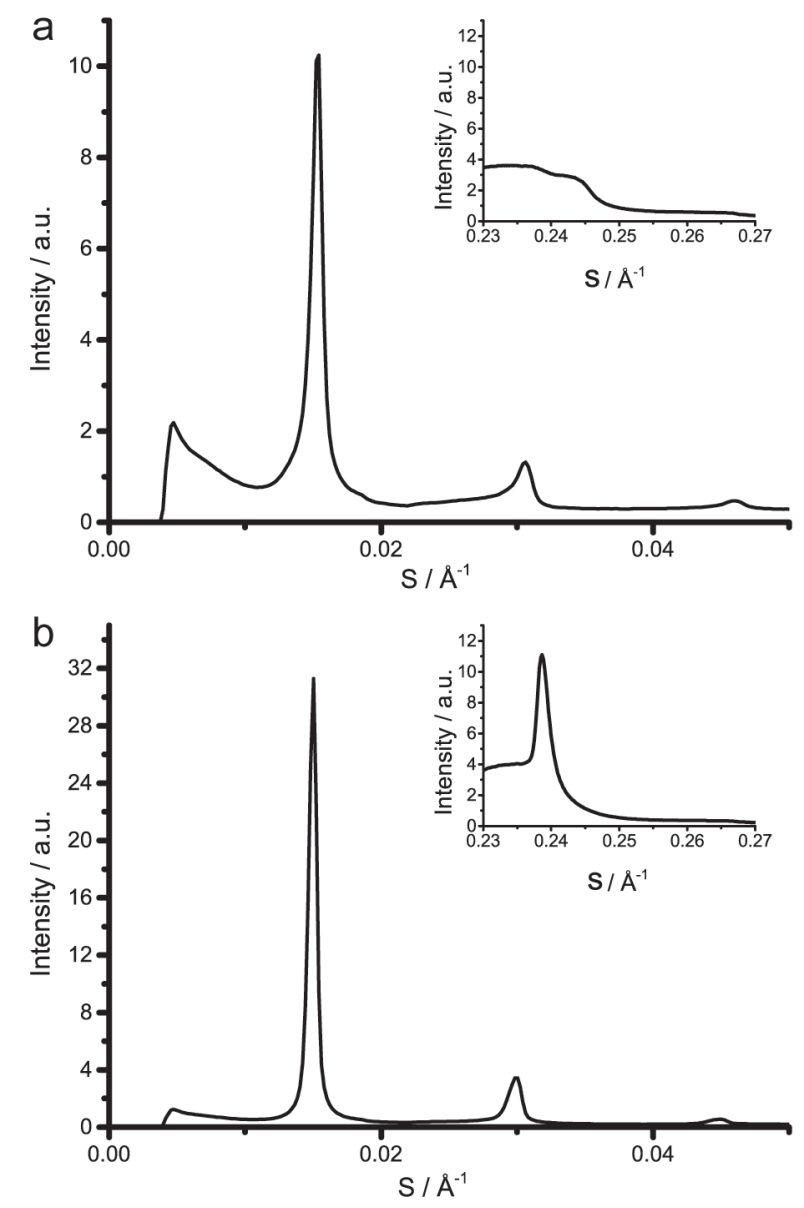

Figure 6. Comparison of (a) $\mathrm{L}_{\beta} 2\left(\mathrm{ESM}: \mathrm{EC} 70: 30 \mathrm{~mol} \%, 30{ }^{\circ} \mathrm{C}\right)$ and (b) $\mathrm{L}_{\beta} 1$ (ESM:EC 60:40 mol\%, $40^{\circ} \mathrm{C}$ ) disrupted gel states showing the characteristic SAXS and WAXS (inset) patterns at atmospheric pressure. 
The $\mathrm{L}_{\beta} 2$ gel is evident in SAXS data from mixtures containing both $40 \mathrm{~mol} \%$ and $60 \mathrm{~mol} \%$ EC, however, above 60 mol\%, pure EC coexists with the mixture and leads to a broad SAXS peak centered at $43 \AA$. This agrees closely with the SAXS peak at $42 \AA$ observed previously in studies of pure EC. ${ }^{4}$

As shown in Figure 2, increasing pressure increases the $\mathrm{L}_{\beta} 2$ to $\mathrm{L}_{\beta} 1$ transition temperature however, pressure has only a very small effect on the lattice parameter of any of the gel phases ( $\sim 1 \AA$ change over the full pressure range studied, Figure 7). Previous experiments have reported an increase in lattice parameter with increasing pressure for pure $\mathrm{ESM}^{7}$ in the fluid phase. Increasing pressure generally increases ordering in lipid hydrocarbon chains ${ }^{25}$ and tends to lead to chain extension an so an increase in lattice parameter. However in a gel phase, the chains have a high degree of conformational order and hence pressure would be expected to have a smaller effect as seen here. 

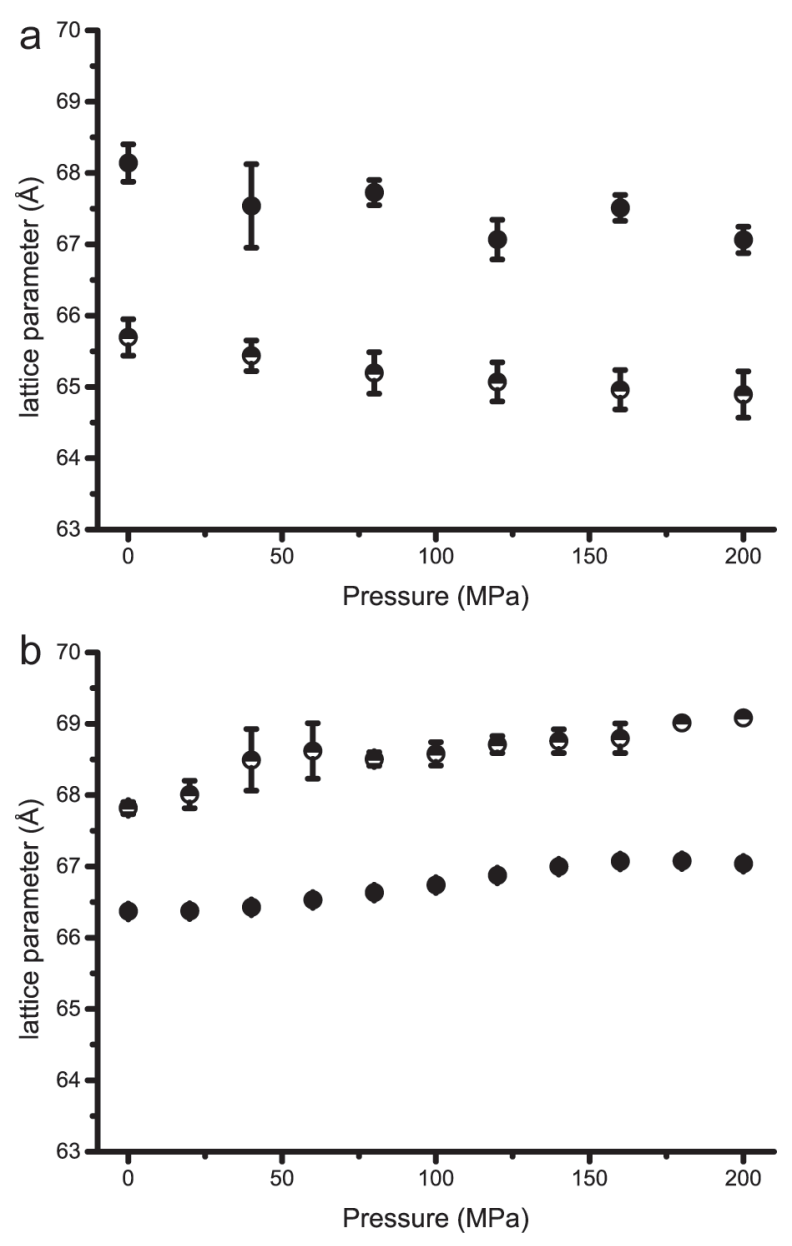

Figure 7. The bilayer spacing (a, lattice parameter) as a function of pressure for (a) ESM:EC 70:30 mol\% showing $\mathrm{L}_{\beta} 2\left(\ominus 30^{\circ} \mathrm{C}\right), \mathrm{P}_{\beta}{ }^{\prime}\left(4^{\circ} \mathrm{C}\right)(\mathrm{b}) \mathrm{ESM}: \mathrm{EC} 40: 60 \mathrm{~mol} \%$ showing $\mathrm{L}_{\beta} 2(\ominus$ $\left.30^{\circ} \mathrm{C}\right), \mathrm{L}_{\beta} 1\left(50^{\circ} \mathrm{C}\right)$. Where error bars are not visible, they are approximately the same size as the datapoints.

Notably in the ESM:EC 70:30 mol\% mixture (Figure 7a) the lattice parameter decreases with increasing pressure, whilst in the $40: 60 \mathrm{~mol} \%$ mixture, (Figure $7 \mathrm{~b}$ ) the lattice parameter increases with increasing pressure. The small decrease in layer spacing observed in both the $\mathrm{L}_{\beta} 2$ and $\mathrm{P}_{\beta}$, in the $70: 30 \mathrm{~mol} \%$ mixture is consistent with previous results for the tilted gel and ripple gel phases in pure $\mathrm{ESM}^{7}$ which has been attributed to a change in lipid chain tilt within the 
bilayer. In the higher EC concentration 40:60 mol\% mixture, the small increase in layer spacing of both the $L_{\beta} 1$ and $L_{\beta} 2$ gel phases with pressure is likely to be due to chain extension as described above and may indicate that the increased EC concentration makes the hydrocarbon chain tilt angle less sensitive to pressure.

\section{Fluid-gel coexistence}

Above its main transition temperature of approximately $39^{\circ} \mathrm{C}$, ESM is known to form a fluid lamellar phase, ${ }^{7}$ however, ceramides are known to have significantly higher gel - fluid transition temperatures. ${ }^{4}$ Phase separation was observed as a doubling of the SAXS peaks in mixtures containing $30 \mathrm{~mol} \% \mathrm{EC}$ at 50 and $60{ }^{\circ} \mathrm{C}$, and for $20 \mathrm{~mol} \% \mathrm{EC}$ at $50^{\circ} \mathrm{C}$ (Figure 8), with the phase transition temperature increasing with pressure as expected. The WAXS data for these mixtures shows a clear, single gel peak characteristic of the $\mathrm{L}_{\beta} 1$ gel phase described above. This phase separation corresponds well to recently demonstrated domain formation at atmospheric pressure in mixed ESM:EC giant unilamellar vesicles (GUVs). ${ }^{26,27}$ Detection of phase separation by SAXS requires long range alignment of domains of each phase, this has been shown to occur in lipid systems ${ }^{28,29}$ and generally phase separation in bulk phases agrees well with domain formation in vesicle systems. 

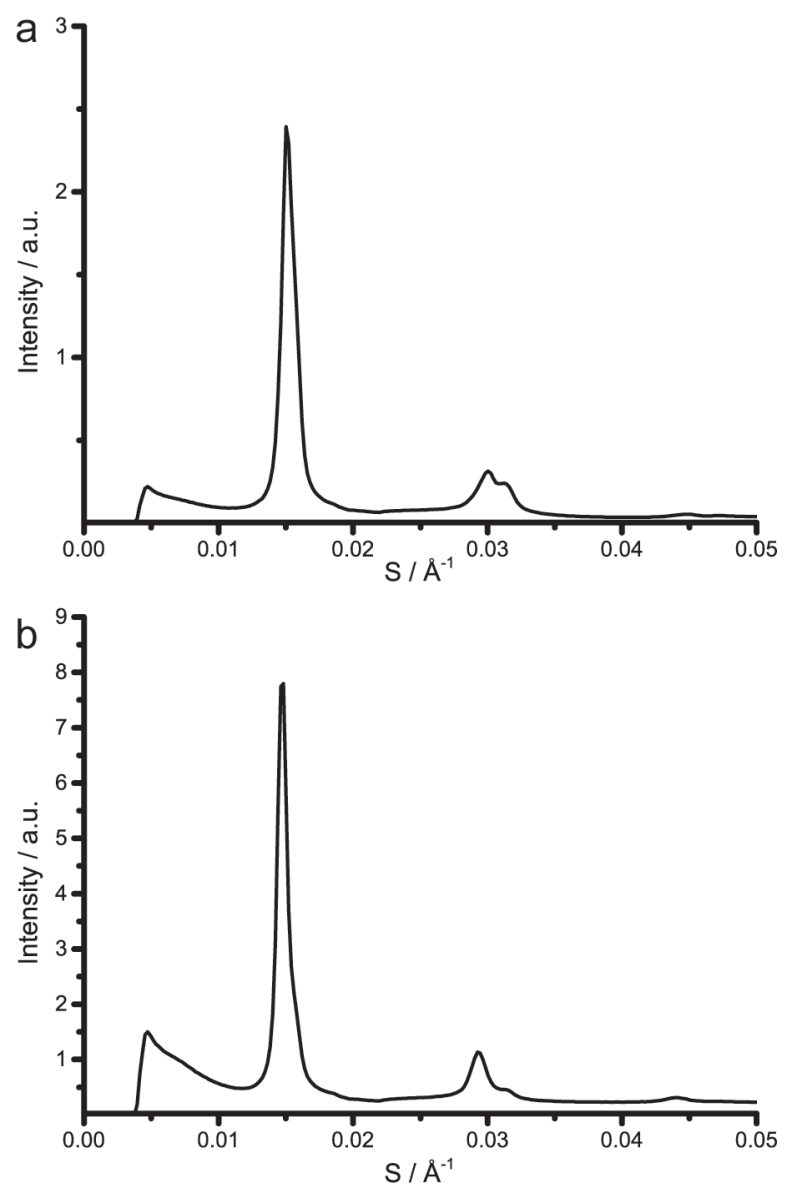

Figure 8. SAXS patterns (atmospheric pressure) showing fluid - gel phase coexistence at $50{ }^{\circ} \mathrm{C}$ for mixtures of ESM : EC (a) 80:20 mol\% (b) $70: 30 \mathrm{~mol} \%$

\section{Fluid Lamellar $\left(L_{\alpha}\right)$}

At EC concentrations of $30 \mathrm{~mol} \%$ and lower, at high temperature the mixtures adopt a single fluid lamellar phase $\left(\mathrm{L}_{\alpha}\right)$. This structure is characterized by sharp evenly spaced peaks in the SAXS pattern and broad scattering in the WAXS region centered on approximately $4.6 \AA$ (ESI Figure S4). The melting temperature of the mixtures lie in between that of the two constituent lipids as expected..$^{30}$ The lattice parameter of ESM at $70^{\circ} \mathrm{C}$ in the fluid lamellar phase is $63.7 \AA$. Increasing the EC content causes an increase in the lattice parameter of up to $1.5 \AA$. 


\section{Hexagonal phase $\left(H_{I I}\right)$}

Above $70{ }^{\circ} \mathrm{C}$ at high EC concentrations (40 and $60 \mathrm{~mol} \%$ ), SAXS data shows that the fluid lamellar phase is replaced by a hexagonal phase in coexistence with a cubic-like structure (Figure 9a). This direct transition between a gel and hexagonal phase is similar to that previously observed in a phosphatidylethanolamine : fatty acid mixture. ${ }^{31}$ It is also possible that a very small region of fluid lamellar phase exists at temperatures in between the gel and hexagonal phases.
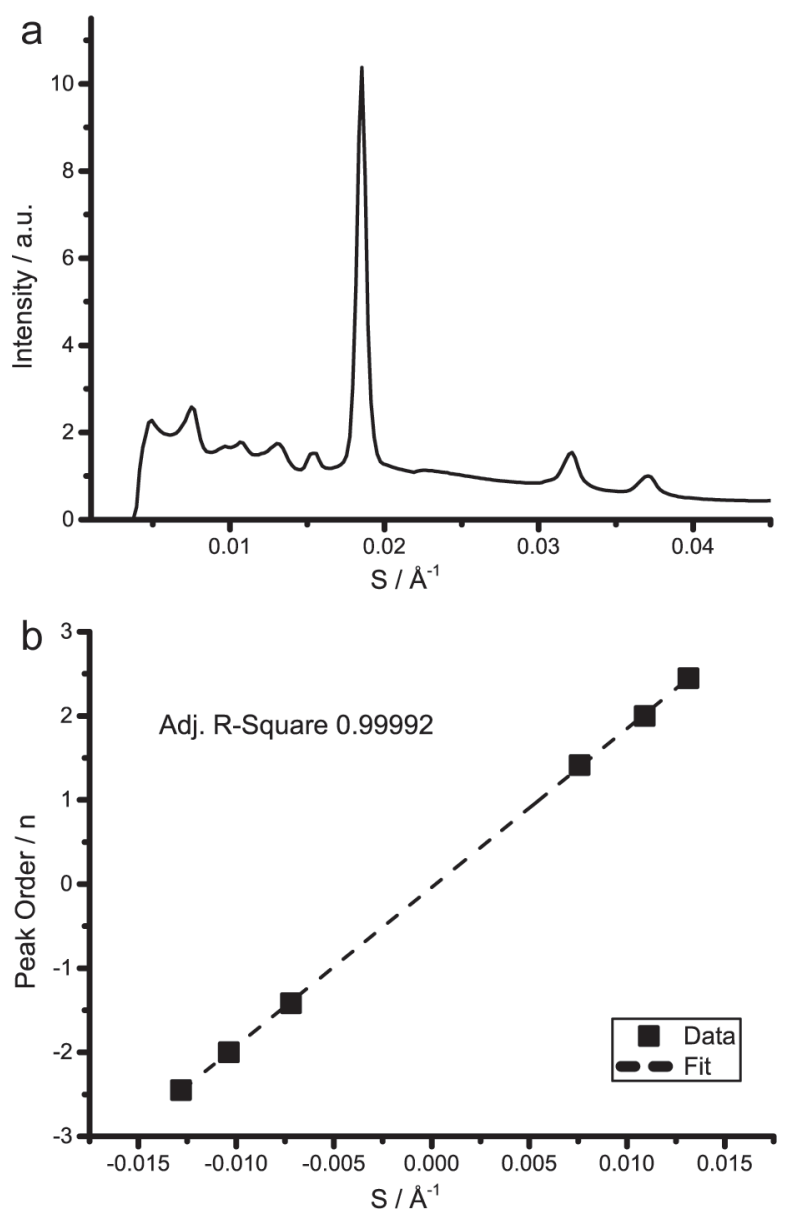

Figure 9. (a) SAXS pattern from ESM:EC 40:60 mol $\%$ at $70{ }^{\circ} \mathrm{C}$ and atmospheric pressure showing (b) First three cubic like diffraction peaks indexed to an Im3m cubic phase. 
The ability of ceramides to promote the formation of inverse hexagonal phases has been reported previously in $\mathrm{EC}^{9,10}$ and this behavior is underpinned by the extremely small headgroup size of ceramide relative to its hydrocarbon chains. However, this is the first example of cubic phase formation in a ceramide mixture. Although the pattern is relatively poorly resolved due to the low fraction of cubic phase and hence intensity, we have reproduced this cubic like structure in numerous samples and synchrotron experiments. In addition, increasing pressure drives the formation of the $L_{\beta} 1$ gel and the cubic-like structure reforms (still in coexistence with the $\mathrm{H}_{\mathrm{II}}$ phase) on returning to atmospheric pressure. We have preliminarily assigned the $\operatorname{Im} 3 \mathrm{~m}$ space

group to this cubic phase, with a lattice parameter of $188 \pm 1 \AA$ (Figure 9b), although we note that more work is needed to characterize this phase fully. It is important to note that both bicontinuous cubic and micellar cubic lipid phases are known with $\operatorname{Im} 3 \mathrm{~m}$ symmetry and either is possible in this case.

\section{CONCLUSIONS}

The pressure-temperature phase behavior of ESM and EC mixtures has been mapped over 30$70{ }^{\circ} \mathrm{C}$ and 0-200 $\mathrm{MPa}$. At temperatures below the ESM transition temperature and low EC compositions, a ripple phase was formed, similar to that seen in pure ESM. This is, to the best of our knowledge the first example of EC mixtures forming a ripple phase. For high EC concentrations, the ripple phase is replaced by a flat gel phase at low temperatures. Above the ESM transition temperature, a different gel phase was observed, characterized by a sharp peak in the WAXS pattern analogous to the EC metastable gel state. At higher temperatures a single fluid lamellar phase was observed similar to that observed in ESM. Notably we do not observe a gel phase analogous to the EC stable gel state; it is likely that the presence of ESM disrupts the 
EC packing and so does not allow formation of the highly ordered EC stable state. At high EC concentrations and high temperatures, a hexagonal phase was observed which coexists with a cubic structure, highlighting the ability of ceramides to modulate membrane curvature.

\section{ACKNOWLEDGMENTS}

This work was supported by EPSRC Programme grant EP/J017566/1, EPSRC Platform grant EP/G00465X/1 and by EPSRC Centre for Doctoral Training Studentships from the Institute of Chemical Biology EP/F500076/1 awarded to Edward Parsons and Nicola McCarthy. We acknowledge Diamond Light Source for provision of synchrotron beamtime and we would like to thank Dr. Andy Smith for assistance using beamline I22.

\section{ASSOCIATED CONTENT}

Supporting Information available: Full DSC data for ESM:EC mixtures. This material is available free of charge via the Internet at http://pubs.acs.org.

\section{AUTHOR INFORMATION}

\section{Corresponding Author}

* nicholas.brooks@imperial.ac.uk

\section{ABBREVIATIONS}

ESM: Egg Sphingomyelin, EC: Egg Ceramide; SAXS: Small Angle X-ray Diffraction, WAXS: Wide Angle X-ray Diffraction 


\section{REFERENCES}

1. Stancevic, B.; Kolesnick, R., Ceramide-rich platforms in transmembrane signaling. FEBS letters 2010, 584 (9), 1728-40.

2. Pettus, B. J.; Chalfant, C. E.; Hannun, Y. A., Ceramide in apoptosis: an overview and current perspectives. Biochimica et biophysica acta 2002, 1585, 114-125.

3. Gulbins, E.; Palmada, M.; Reichel, M.; Luth, A.; Bohmer, C.; Amato, D.; Muller, C. P.; Tischbirek, C. H.; Groemer, T. W.; Tabatabai, G.; Becker, K. A.; Tripal, P.; Staedtler, S.; F, A. T.; van Brederode, J.; Alzheimer, C.; Weller, M.; E, L. U.; Kleuser, B.; Grasme, H.; Kornhuber, J., Acid sphingomyelinase-ceramide system mediates effects of antidepressant drugs. Nature Medicine 2013, 19, 934-938.

4. Shah, J.; Atienza, J. M.; Duclos, R. I.; Rawlings, A. V.; Dong, Z.; Shipley, G. G., Structural and thermotropic properties of synthetic C16:0 (palmitoyl) ceramide: Effect of hydration. Journal of lipid research 1995, 36, 1936-1944.

5. Do, U. H.; Ramachandran, S., mild alkali stable phospholipids in chicken egg yolks. Journal of lipid research 1980, 21, 888-894.

6. Filippov, A.; Oradd, G.; Lindblom, G., Sphingomyelin structure influences the lateral diffusion and raft formation in lipid bilayers. Biophysical journal 2006, 90 (6), 2086-92.

7. Shaw, K. P.; Brooks, N. J.; Clarke, J. A.; Ces, O.; Seddon, J. M.; Law, R. V., Pressuretemperature phase behaviour of natural sphingomyelin extracts. Soft Matter 2012, 8 (4), 1070.

8. Pinto, S. N.; Silva, L. C.; Futerman, A. H.; Prieto, M., Effect of ceramide structure on membrane biophysical properties: the role of acyl chain length and unsaturation. Biochimica et biophysica acta 2011, 1808 (11), 2753-60.

9. Sot, J.; Aranda, F. J.; Collado, M. I.; Goni, F. M.; Alonso, A., Different effects of longand short-chain ceramides on the gel-fluid and lamellar-hexagonal transitions of phospholipids: a calorimetric, NMR, and x-ray diffraction study. Biophysical journal 2005, 88 (5), 3368-80.

10. Veiga, M. P.; Arrondo, J. L. R.; Goni, F. M.; Alonso, A., Ceramides in phospholipid membranes: effects on bilayer stability and transition to nonlamellar phases. Biophysical journal 1999, 76, 342-350.

11. Jimenez-Rojo, N.; Garcia-Arribas, A. B.; Sot, J.; Alonso, A.; Goni, F. M., Lipid bilayers containing sphingomyelins and ceramides of varying $\mathrm{N}$-acyl lengths: a glimpse into sphingolipid complexity. Biochimica et biophysica acta 2014, 1838 (1 Pt B), 456-64.

12. Artetxe, I.; Sergelius, C.; Kurita, M.; Yamaguchi, S.; Katsumura, S.; Slotte, J. P.; Maula, T., Effects of sphingomyelin headgroup size on interactions with ceramide. Biophysical journal 2013, 104 (3), 604-12.

13. Lonnfors, M.; Langvik, O.; Bjorkbom, A.; Slotte, J. P., Cholesteryl phosphocholine--a study on its interactions with ceramides and other membrane lipids. Langmuir 2013, 29 (7), 2319-29.

14. Busto, J. V.; Fanani, M. L.; De Tullio, L.; Sot, J.; Maggio, B.; Goni, F. M.; Alonso, A., Coexistence of immiscible mixtures of palmitoylsphingomyelin and palmitoylceramide in monolayers and bilayers. Biophysical journal 2009, 97 (10), 2717-26.

15. Sot, J.; Bagatolli, L. A.; Goni, F. M.; Alonso, A., Detergent-resistant, ceramide-enriched domains in sphingomyelin/ceramide bilayers. Biophysical journal 2006, 90 (3), 903-14.

16. Castro, B. M.; Silva, L. C.; Fedorov, A.; de Almeida, R. F.; Prieto, M., Cholesterol-rich fluid membranes solubilize ceramide domains: implications for the structure and dynamics of 
mammalian intracellular and plasma membranes. The Journal of biological chemistry 2009, 284 (34), 22978-87.

17. Staneva, G.; Chachaty, C.; Wolf, C.; Koumanov, K.; Quinn, P. J., The role of sphingomyelin in regulating phase coexistence in complex lipid model membranes: competition between ceramide and cholesterol. Biochimica et biophysica acta 2008, 1778 (12), 2727-39.

18. Megha; London, E., Ceramide selectively displaces cholesterol from ordered lipid domains (rafts): implications for lipid raft structure and function. The Journal of biological chemistry 2004, 279 (11), 9997-10004.

19. Ramirez, D. M.; Jakubek, Z. J.; Lu, Z.; Ogilvie, W. W.; Johnston, L. J., Changes in order parameters associated with ceramide-mediated membrane reorganization measured using pTIRFM. Langmuir 2013, 29 (51), 15907-18.

20. Brooks, N. J.; Gauthe, B. L.; Terrill, N. J.; Rogers, S. E.; Templer, R. H.; Ces, O.; Seddon, J. M., Automated high pressure cell for pressure jump x-ray diffraction. The Review of scientific instruments 2010,81 (6), 064103.

21. Heron, A. Stored Curvature Elastic Stress in Lipid Membranes and its Effect upon the Activity of CTP:Phosphocholine Cytidylyltransferase. PhD Thesis, Imperial College London (University of London), 2006.

22. Leidy, C.; Kaasgaard, T.; Crowe, J. H.; Mouritsen, O. G.; Jorgensen, K., Ripples and the formation of anisotropic lipid domains: imaging two-component supported double bilayers by atomic force microscopy. Biophysical journal 2002, 83 (5), 2625-33.

23. Carrer, D. C.; Maggio, B., Phase behaviour and molecular interactions in mixtures of ceramide with dipalmitoylphosphatidylcholine. Journal of lipid research 1999, 40, 1978-1989.

24. Metcalf, R.; Pandit, S. A., Mixing Properties of Sphingomyelin Ceramide Bilayers: A Simulation Study. J Phys Chem B 2012, 116 (15), 4500-4509.

25. Brooks, N. J.; Ces, O.; Templer, R. H.; Seddon, J. M., Pressure effects on lipid membrane structure and dynamics. Chemistry and physics of lipids 2011, 164 (2), 89-98.

26. Busto, J. V.; Garcia-Arribas, A. B.; Sot, J.; Torrecillas, A.; Gomez-Fernandez, J. C.; Goni, F. M.; Alonso, A., Lamellar gel (lbeta) phases of ternary lipid composition containing ceramide and cholesterol. Biophysical journal 2014, 106 (3), 621-30.

27. Castro, B. M.; Prieto, M.; Silva, L. C., Ceramide: a simple sphingolipid with unique biophysical properties. Progress in lipid research 2014, 54, 53-67.

28. Tayebi, L.; Ma, Y.; Vashaee, D.; Chen, G.; Sinha, S. K.; Parikh, A. N., Long-range interlayer alignment of intralayer domains in stacked lipid bilayers. Nature materials 2012, 11 (12), 1074-80.

29. Uppamoochikkal, P.; Tristram-Nagle, S.; Nagle, J. F., Orientation of tie-lines in the phase diagram of DOPC/DPPC/cholesterol model biomembranes. Langmuir 2010, 26 (22), 17363-8.

30. Veatch, S. L.; Keller, S. L., Separation of Liquid Phases in Giant Vesicles of Ternary Mixtures of Phospholipids and Cholesterol. Biophysical journal 2003, 85, 3074-3083.

31. Marsh, D.; Seddon, J. M., Gel to inverted hexagonal phase transitions in phosphatidylethanolamines and fatty acid phosphatidylcholine mixtures demonstrated by 31P NMR spectroscopy and x-ray diffration. Biochimica et biophysica acta 1982, 25 (690 (1)), 117123. 

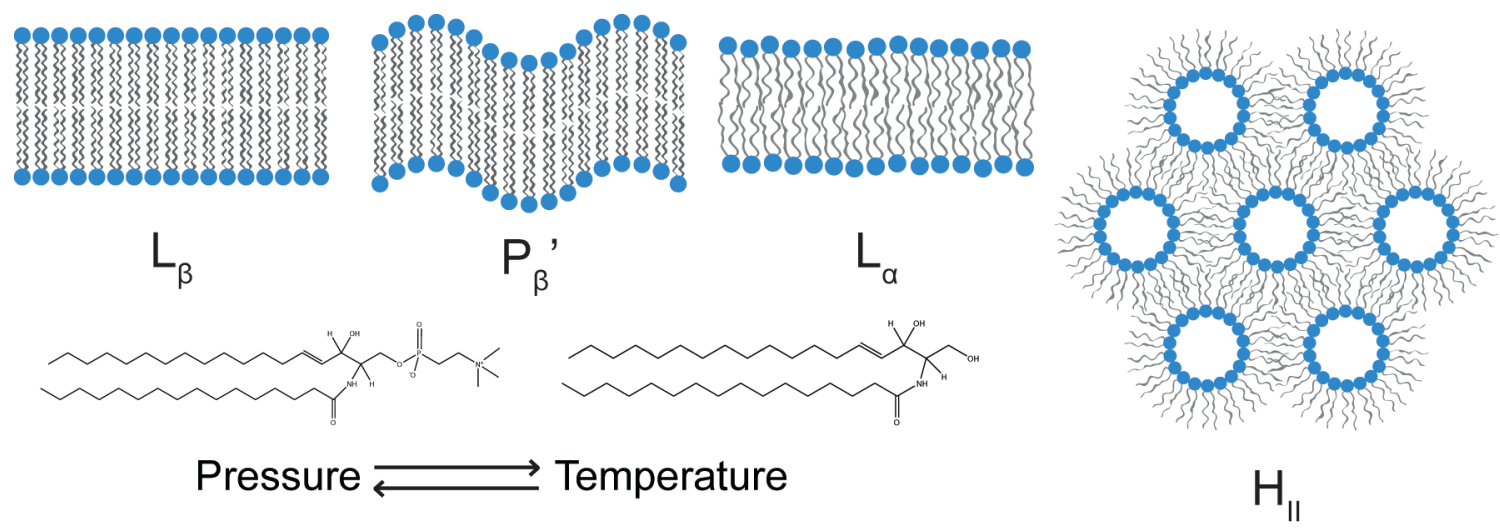